\title{
On Stability Equilibrium Analysis of Endemic Malaria
}

\author{
${ }^{1}$ I.I Raji, ${ }^{1}$ A.A.Abdullahi and ${ }^{2}$ M.O Ibrahim \\ ${ }^{I}$ Department of Mathematics and Statistics, Federal Polytechnic, Ado-Ekiti, Nigeria. \\ ${ }^{2}$ Department of Mathematics, University of Ilorin, Ilorin, kwara state, Nigeria.
}

\begin{abstract}
This paper considers the stability equilibrium of malaria in a varying population. We established the Disease free equilibrium and the endemic equilibrium and carried out the stability analysis of the Disease free equilibrium state (the state of complete eradication of malaria from the population).
\end{abstract}

Keywords: malaria, mathematical model, disease-free equilibrium, endemic equilibrium, basic reproduction number.

\section{Introduction}

Several insects are known to be vectors of human disease. Mosquitoes in particular enjoy the questionable honour of having been the first insects to be associated with the transmission of a disease.

Malaria is an infectious disease caused by the Plasmodium parasite and transmitted between mosquitos.In 1898, the Italian Zoologist G.B. Grassi and his co-workers first described the complete cycle of the human malaria parasites and pointed to a species of the genus Anopheles as responsible of malaria transmission. Of more than 480 species of anopheles, only about 50 species transmit malaria. The habits of most of the anopheles mosquitoes have been characterized as anthropophagic (prefer human blood meal), endothermic (bite indoors), and nocturnal (bite at night) with peak biting at midnight, between $11 \mathrm{pm}$ and $2 \mathrm{am}$. The epidemiology of malaria in a given environment is the result of a complex interplay among man, plasmodia, and anopheline mosquitoes. These three elements have to be present for malaria transmission to occur in nature. The incidence of malaria has been growing recently due to increasing parasite drug- resistance and mosquito insecticide resistance.

It remains one of the most difficult epidemiological, pharmacological and immunological challenges in sub-Sahara Africa. Its influence has been profound and sustained over the centuries and malaria remains a major cause of morbidity and mortality. Moreover, the epidemiological situation appears to be changing for the worse in many countries (Offosu, Amaah, 1991).

An estimated $40 \%$ of the world's populations live in malaria endemic areas. Each year 300 to 500 million people develop malaria. It kills about 700,000 to 2.7 million people a year $75 \%$ of whom are Africa children

Malaria is the ninth largest cause of death and disability globally.Malaria episodes lead to direct cost associated with health care and health care - related transport and indirect costs associated with lost income for patients, lost income for careers and school days missed by children.

In areas where malaria transmissions are high, the highest number of cases is concentrated among young children. However, malaria illness affects all age groups which leads to a higher loss of income and decreased productivity [8].

We develop a mathematical model to better understand the transmission and spread of malaria. This model is used to determine which factors are most responsible for the spread of malaria.

\section{Model Formulation}

We formulate a model similar to that of $[11,12]$ describing the transmission of malaria.The equations for model is as described below

$$
\begin{gathered}
\frac{d S_{h}}{d t}=P+\varepsilon R_{h}-\left(\delta_{h}+\mu_{h}\right) S_{h} \\
\frac{d E_{h}}{d t}=\delta_{h} S_{h}-\left(\mu_{h}+i_{h}\right) E_{h} \\
\frac{d I_{h}}{d t}=i_{h} E_{h}-\left(\mu_{h}+\eta_{h}\right) I_{h}-q I_{h} \\
\frac{d R_{h}}{d t}=q I_{h}-\left(\mu_{h}+\varepsilon\right) R_{h}
\end{gathered}
$$




$$
\begin{gathered}
\frac{d S_{v}}{d t}=V-\left(\psi_{v}+\delta_{v}\right) S_{v} \\
\frac{d E_{v}}{d t}=\delta_{v} S_{v}-\left(\psi_{v}+i_{v}\right) E_{v} \\
\frac{d I_{v}}{d t}=i_{v} E_{v}-\psi_{v} I_{v}
\end{gathered}
$$

Table 1: The state variables for the malaria model equation 1.0

Parameter

$S_{h}(t)$

$E_{h}(t)$

$I_{h}(t)$

$R_{h}(t)$

$S_{v}(t)$

$E_{v}(t)$

$N_{h}(t)$

$N_{v}(t)$
Description

Number of susceptible human hosts at time $t$

Number of exposed human hosts at time $t$

Number of infections human hosts at time $t$

Number of recovered human with temporary immunity of time $t$

Number of susceptible mosquito vectors at time $t$

Number of exposed mosquito vectors at time $t$

Total human population

\begin{tabular}{|c|c|}
\hline Parameter & Description \\
\hline $\mathrm{P}$ & Birth \\
\hline$\mu_{h}$ & Natural death rate for humans \\
\hline$\delta_{h}$ & Force of infection for humans from susceptible state to exposed state. \\
\hline$i_{h}$ & Incident rate for humans \\
\hline $\mathrm{Q}$ & Treatment of infected humans \\
\hline$\eta_{h}$ & Disease- induced death rate for humans \\
\hline$\varepsilon$ & Rate of loss of immunity for humans \\
\hline $\mathrm{V}$ & Recruitment rate of mosquitoes \\
\hline$\delta_{v}$ & Force of infection of mosquitoes from susceptible state to exposed state. \\
\hline$i_{v}$ & Incident rate for mosquitoes \\
\hline$\varphi_{v}$ & Natural death rate for mosquitoes \\
\hline$\Lambda_{v h}$ & $\begin{array}{l}\text { Probability of transmission of infection from an infectious mosquito to a susceptible human provided there is a } \\
\text { contact. }\end{array}$ \\
\hline$\Lambda_{h v}$ & $\begin{array}{l}\text { Probability of transmission of infection from an infectious human to a susceptible mosquitoes provided there is } \\
\text { a contact }\end{array}$ \\
\hline
\end{tabular}

Total mosquito population

Table II: Model parameters and their interpretations for the malaria model equation 1.0

TOTAL POPULATION DENSITY

The total population sizes are

$$
\begin{gathered}
N_{h}=S_{h}+E_{h}+I_{h}+R_{h} \\
N_{v}=S_{v}+E_{v}+I_{v} \\
\frac{d N_{h}}{d t}=P-\mu_{h} N_{h}-\eta_{h} I_{h} \\
\operatorname{also} \frac{d N_{v}}{d t}=V-\varphi_{v} N_{v} \\
\text { where } \delta_{h}=\Lambda_{v h}, \delta_{v}=\Lambda_{h v}
\end{gathered}
$$

where $A_{v h}$ denotes the rate at which the susceptible human $\mathrm{S}_{\mathrm{h}}$, become infected by infectious female Anopheles mosquitoes $\mathrm{I}_{\mathrm{v}}$ and $\Lambda_{h v}$ represents the rate at which the susceptible mosquitoes $\mathrm{S}_{\mathrm{v}}$ are infected by infectious human $I_{h}$.

The model is analysed to examine the critical factors which determine the persistence or eradication of malaria. We hence determine if the mode is well posed.

Theorem (2.1): The solution of (2.0) are feasible for all $t>0$ and is defined in the subset $\Omega \times[0, \infty)$ of $R_{+}^{7}$ 
Proof:

Let $\Omega_{h}=\left(S_{h}, E_{h}, I_{h}, R_{h}, S_{v}, E_{v}, I_{v}\right) \in R_{+}^{7}$ be any solution of the system (2.0) with non-negative initial conditions.

In absence of the disease (malaria), $I_{h}=0$,

$$
\begin{gathered}
\frac{d N_{h}}{d t} \leq P-\mu_{h} N_{h} \\
\frac{d N_{h}}{d t}+\mu_{h} N_{h} \leq P \\
\frac{d}{d t}\left(N_{h} e^{\mu_{h} t}\right) \leq P e^{\mu_{h} t} \\
N_{h} e^{\mu_{h} t} \leq \frac{P e^{\mu_{h} t}}{\mu_{h}}+c
\end{gathered}
$$

Where $\mathrm{c}$ is a constant of integration

$$
\begin{array}{r}
N_{h} \leq \frac{P}{\mu_{h}}+c e^{\mu_{h} t} \\
\text { at } \mathrm{t}=0 \\
N_{h}(0) \leq \frac{P}{\mu_{h}}+c \\
N_{h}(0) \leq \frac{P}{\mu_{h}} \leq c \\
\text { With } \\
N_{h} \leq \frac{P}{\mu_{h}}+\left(N_{h}(0)-\frac{P}{\mu_{h}}\right) e^{-\mu_{h} t} \\
0 \leq N_{h} \leq \frac{P}{\mu_{h}} a t t \rightarrow \infty
\end{array}
$$

Therefore, as $t \rightarrow \infty$ in (2.2), the human population.

$N_{h}$ approaches $\frac{P}{\mu_{h}}$, where $\frac{P}{\mu_{h}}=k$ Called the carrying capacity.

Hence all feasible solution set of the human of the human population of the model (2.1) enter the region.

$$
\Omega_{h}=\left\{\left(S_{h}, E_{h}, I_{h}, R_{h}\right) \in \mathbb{R}_{+}^{4}: S_{h}>0, E_{h} \geq 0, I_{h} \geq 0, R_{h} \geq 0 \text { and } N_{h} \leq \frac{P}{\mu_{h}}\right\}
$$

In like manner, the feasible solutions set of the mosquito population enters the region

$$
\begin{gathered}
\Omega_{v}=\left\{\left(S_{v}, E_{v}, I_{v}\right) \epsilon \mathbb{R}_{+}^{3}: S_{v}>0, E_{v} \geq 0, I_{v} \geq 0, N_{v} \geq 0 a \leq \frac{V}{\varphi_{v}}\right\} \\
\frac{d S_{h}}{d x}=P+\varepsilon R_{h}-\delta_{h} S_{h}-\mu_{h} S_{h} \geq-\delta_{h} S_{h}-\mu_{h} S_{h} \\
\frac{d S_{h}}{d x}-\left(\delta_{h} S_{h}+\mu_{h} S_{h}\right) \geq-\left(\delta_{h} S_{h}-\mu_{h}\right) S_{h} \\
\frac{d S_{h}}{S_{h}} \geq-\left(\delta_{h}+\mu_{h}\right) d t \\
d u S_{h} \geq-\left(\delta_{h}+\mu_{h}\right) t+C_{1} \\
S_{h} \geq e^{-\left(\delta_{h}+\mu_{h}\right) t+C_{1}} \geq e^{-\left(\delta_{h}+\mu_{h}\right)} e^{C_{1}} \\
S_{h}(t) \geq A e^{-\left(\delta_{h}+\mu_{h}\right) t} \\
A t t=0 \\
S_{h}(0) \geq A \\
S_{h}(0) e^{-\left(\delta_{h}+\mu\right) t} \geq 0 \\
\therefore S_{h}(t) \geq S_{h}(0) e^{-\left(\delta_{h}+\mu\right) t} \geq 0
\end{gathered}
$$

Also the remaining equations of system (2.0) are all positive fort $>0$.

Hence, the domain $\Omega$ is positively invariant, because no solution paths leave through any boundary. Since paths cannot leave $\Omega$, solutions exist for all positive time. Thus the model is mathematically and epidemiologically well-posed.

Disease free equilibrium: We now solve the model equations to obtain the equilibrium states. At the equilibrium state in the absence of the disease, we have $E_{h}=I_{h}=E_{v}=I_{v}=0$.

From (1.0)

$$
\mathrm{P}-\mu_{h}-N_{h}=0
$$




$$
\begin{aligned}
& N_{h}^{*}=\frac{P}{\mu_{h}} \\
& N_{v}^{*}=\frac{V}{\varphi_{v}}
\end{aligned}
$$

Therefore, the disease free equilibrium point of the malaria mode (3.2) is $E_{o}=\left(S_{h}, E_{h}, I_{h}, R_{h}, S_{v}, E_{v}, I_{v}\right)=$ $\left(\frac{P}{\mu_{h}}, 0,0,0, \frac{V}{\psi_{v}}, 0,0\right)$.

This is the state in which there is no infection (in the absence of malaria) in the society.

\section{THE BASIC REPRODUCTION RATIO $R_{o}$}

This is the expected number of secondary cases, produced, in a completely susceptible population, by a typical infected individual during its entire period of infectiousness, and mathematically as the dominant eigen value of positive linear operator.

In the original parameters $R_{o}=\sqrt{\frac{V i_{h} i_{v} \Lambda_{h v} \Lambda_{v h} \mu}{P \psi\left(i_{h}+\mu\right)(q+\mu+\eta)\left(i_{v}+\psi\right) \psi}}$

\section{LOCAL STABILITY OF THE DISEASE-FREE EQUILIBRIUM}

Theorem 3.1: The disease-free equilibrium point is locally asymptotically stable if $R_{o}<1$ and is unstable if $R_{o}>1$.

Proof: The matrix of the system of equation is

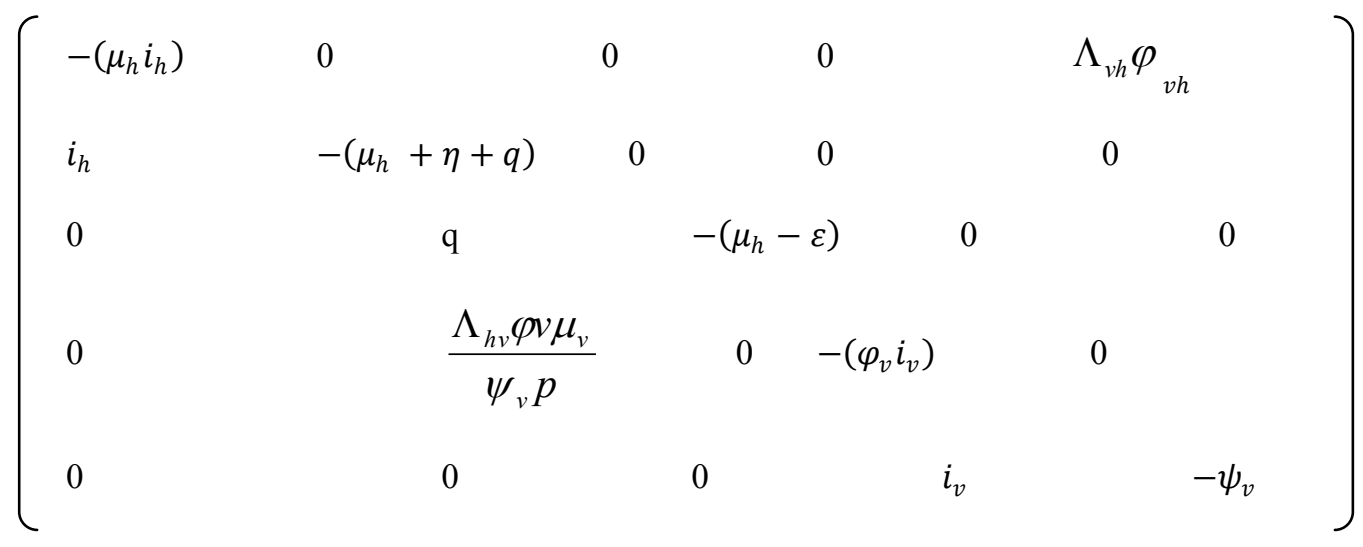

The Jacobian matrix is given as $(A-\lambda I)$

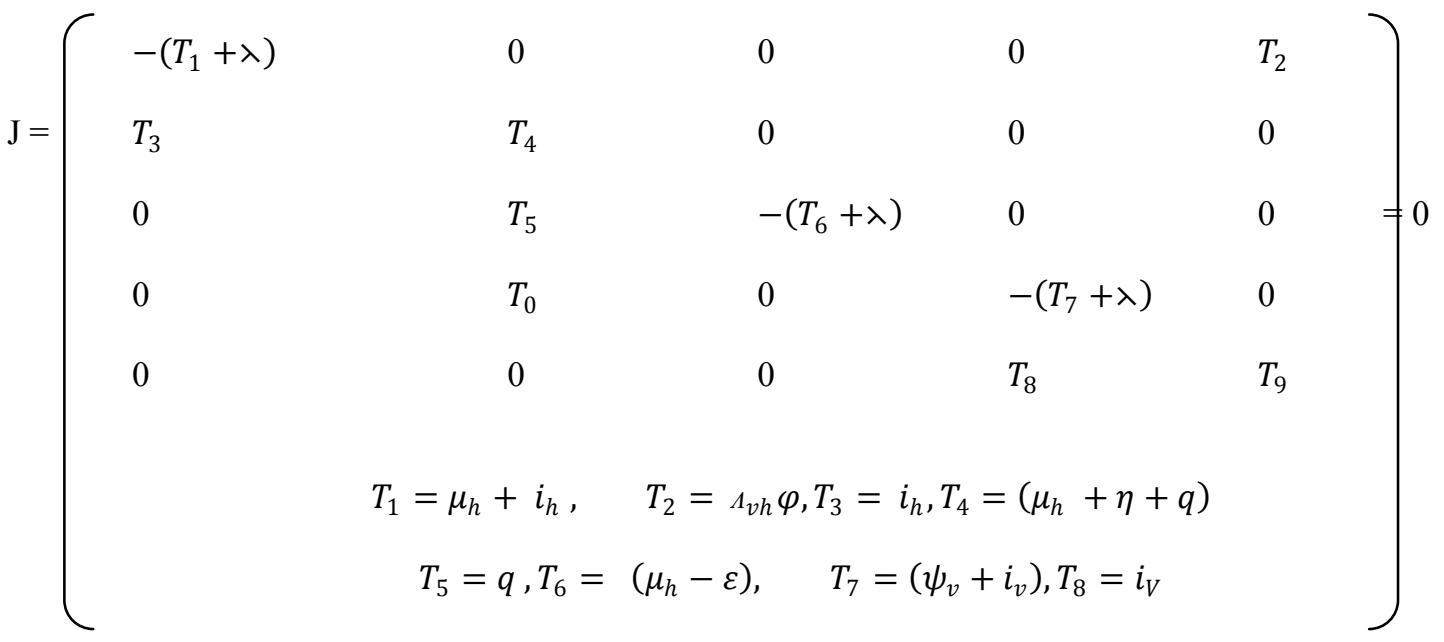

$T_{9}=\psi_{v}, T_{0}=\frac{\Lambda_{h v} \varphi v \mu_{v}}{\psi_{v} p}$

From the third column, which has a diagonal entry, one of the eigenvalues is $-\left(T_{6}+\lambda\right)$.

The matrices therefore reduces to 
$J=\left|\begin{array}{clll}-\left(T_{1}+\lambda\right) & 0 & 0 & T_{2} \\ T_{3} & -\left(T_{4}+\lambda\right) & 0 & 0 \\ 0 & T_{0} & -\left(T_{7}+\lambda\right) & 0 \\ 0 & 0 & T_{8} & -\left(T_{9}+\lambda\right)\end{array}\right|=0$

which forms the characteristics equation

$$
\begin{gathered}
\left(T_{1}+\lambda\right)\left(T_{4}+\lambda\right)\left(T_{7}+\lambda\right)\left(T_{9}+\lambda\right)-T_{2} T_{3} T_{8} T_{0}=0 \\
=\left(T_{1} T_{4}+\lambda T_{1}+\lambda T_{4}+\lambda^{2}\right)\left(T_{7} T_{9}+\lambda T_{7}+\lambda T_{9}+\lambda^{2}\right)-T_{2} T_{3} T_{8} T_{0}=0 \\
\lambda^{4}+\lambda^{3} \gamma_{1}+\lambda^{2} \gamma_{2}+\lambda \gamma_{3}+\gamma_{0}=0
\end{gathered}
$$

For

$$
\begin{aligned}
& \gamma_{1}=T_{1}+T_{4}+T_{7}+T_{9} \\
& \gamma_{2}=T_{1} T_{7}+T_{1} T_{9}+T_{4} T_{9}+T_{7} T_{9}+T_{1} T_{4} \\
& \gamma_{3}=T_{1} T_{4} T_{7}+T_{1} T_{4} T_{9}+T_{1} T_{7} T_{9}+T_{4} T_{7} T_{9}+T_{4} T_{7} \\
& \gamma_{0}=T_{1} T_{4} T_{7} T_{9}-T_{2} T_{3} T_{8} T_{0}
\end{aligned}
$$

To evaluate the signs of the roots of, we use the Routh - Hurwitz criterion and Descartes' Rule of sign.

\section{Theorem 3.2: $\quad$ Routh-Hurwitz Criteria}

$$
\text { Given the polynomial }
$$

$$
P(\lambda)=\lambda^{n}+\gamma_{1} \lambda^{n-1}+\ldots \gamma_{n-1} \lambda+\gamma_{n}
$$

where the coefficients $\gamma_{i}$ are real constants, $i=1, \ldots, n$; define the $\mathrm{n}$ Hurwitz matrices is equal to the number of sign changes $\gamma_{i}$ of the characteristic polynomial.

$$
F_{o}=1, \quad F_{1}=\gamma_{1}, \quad F_{2}=\left|\begin{array}{cc}
\gamma_{1} & 1 \\
\gamma_{3} & \gamma_{2}
\end{array}\right|, F_{3}=\left|\begin{array}{ccc}
\gamma_{1} & 1 & 0 \\
\gamma_{3} & \gamma_{2} & \gamma_{1} \\
\gamma_{5} & \gamma_{4} & \gamma_{3}
\end{array}\right|, F_{4}=\left|\begin{array}{llll}
\gamma_{1} & 1 & 0 & 0 \\
\gamma_{3} & \gamma_{2} & \gamma_{1} & 1 \\
\gamma_{5} & \gamma_{4} & \gamma_{3} & \gamma_{2}
\end{array}\right|
$$

where $\gamma_{i}=0$ ifi $>n$. All of the roots of the polynomial $P(\lambda)$ are negatives or have negative real parts if and only if the determinants of all Hurwitz matrices are positive: $\operatorname{det}\left(F_{j}\right)>0, j=1,2, \ldots ., n$.

We show that when $R_{o}<1$, all the coefficients, $\gamma_{i}$, of the characteristics equation and $F_{j}$ are positive.

From the characteristic equation, with $\mathrm{n}=4$, the Routh - Hurwitz criteria are $\gamma_{1}>0, \gamma_{2}>0, \gamma_{3}>0, \gamma_{4}>0$ anddet $\left(F_{1}\right)=\gamma_{1}>0$,

$$
\begin{gathered}
\operatorname{det}\left(F_{2}\right)=\left|\begin{array}{ll}
\gamma_{1} & 1 \\
0 & \gamma_{2}
\end{array}\right|=\gamma_{1} \gamma_{2}>0, \\
\operatorname{det}\left(F_{3}\right)=\left|\begin{array}{ccc}
\gamma_{1} & 1 & 0 \\
\gamma_{3} & \gamma_{2} & \gamma_{1} \\
0 & 0 & \gamma_{3}
\end{array}\right| \\
=\gamma_{1} \gamma_{2} \gamma_{3}-\gamma_{3}^{2}>0 \\
\text { i.e. }=\gamma_{1} \gamma_{2}-\gamma_{3}>0
\end{gathered}
$$

and

$$
\begin{aligned}
& =\gamma_{1} \gamma_{2} \gamma_{3}-\gamma_{1}^{2} \gamma_{4}-\gamma_{3}^{2}>0 \\
& =\gamma_{1} \gamma_{2} \gamma_{3}-\gamma_{3}^{2}-\gamma_{1}^{2} \gamma_{4}>0
\end{aligned}
$$

Since all the determinants of the Hurwitz are positive, which implies that all the Eigen values of the Jacobian matrix have negative real part. Hence disease -free equilibrium point is asymptotically stable and $R_{o}>1$. 


\section{The Endemic Equilibrium Point}

Endemic equilibrium points are steady state situations where the disease persist in the population (all state variables are positive). The endemic equilibrium of the model is given as $E^{*}=\left(S_{h}, E_{h}, I_{h}, R^{*}, S_{v}, E^{*}, I_{v}\right)>0$.

Consider equałion $* *$

$$
\begin{gathered}
\frac{d S_{h}}{d t}=P+\varepsilon R_{h}-\frac{\Lambda_{v h} \varphi I_{v} S_{h}}{N_{h}}-\mu S_{h} \\
\frac{d E_{h}}{d t}=\frac{\Lambda_{v h} \varphi I_{v} S_{h}}{N_{h}}-\mu_{h} E_{h}-i_{h} E_{h}=0 \\
\frac{d I_{h}}{d t}=i_{h} E_{h}-\left(\mu_{h}+\Lambda_{h}\right) I_{h}-q I_{h}=0 \\
\frac{d R_{h}}{d t}=q I_{h}-\mu_{h} R_{h}-\varepsilon R_{h}=0 \\
\frac{d S_{v}}{d t}=v-\psi_{v} S_{v}-\frac{\Lambda_{h v} \varphi I_{h} S_{v}}{N_{h}} \\
\frac{d E_{v}}{d t}=\frac{\Lambda_{h v} \varphi I_{h} S_{v}}{N_{h}}-\psi_{v} E_{v}-i_{v} E_{v}=0 \\
\frac{d I_{v}}{d t}=i_{v} E_{v}-\psi_{v} E_{v}=0 \\
\text { from } \\
i_{v} E_{v}-\psi_{v} I_{v}=0 \\
\psi_{v} I_{v}=i_{v} E_{v}
\end{gathered}
$$

Also

$$
E_{v}\left(\psi_{v}+i_{v}\right)=\frac{\Lambda_{h v} \varphi I_{h} S_{v}}{N_{h}}
$$$$
E_{v}^{*}=\frac{\Lambda_{h v} \varphi I_{h} S_{v}}{N_{h}\left(\psi_{v}+i_{v}\right)}
$$$$
\text { Substitute (ii) and (i) }
$$$$
I_{v}^{*}=\frac{\Lambda_{h v} \varphi I_{h} S_{v}}{\psi_{v} N_{h}\left(\psi_{v}+i_{v}\right)}
$$$$
\text { from } \frac{d S_{v}}{d t}=v-\psi_{v} S_{v}-\frac{\Lambda_{h v} \varphi I_{h} S_{v}}{N_{h}}=0
$$$$
S_{v}\left(\psi_{v}+\frac{\Lambda_{h v} \varphi I_{v}}{N_{h}} \frac{{ }_{\hat{\varphi} h h} \varphi I_{v} S_{h}}{\mu_{h}}\right)=v
$$$$
S_{v}^{*}=\frac{v N_{h}}{N_{h} \psi_{v}+\Lambda_{h v} \varphi I_{v}}
$$$$
\text { Substitute (iv) in (iii) }
$$$$
I_{v}^{*}=\frac{i_{v} \Lambda_{h v} \varphi I_{h} v N_{h}}{\psi_{v} N_{h}\left(\psi_{v}+i_{v}\right)\left(N_{h} \psi_{v}+\Lambda_{h v} \varphi I_{v}\right)}
$$

\section{Conclusion}


From our analysis, we found that the disease free equilibrium is stable when the threshold parameter is less than unity. However, the disease could be reduced if the contact rate is avoided. This could be achieved by the use of insecticide bed treated net and or indoor spraying..

\section{References}

[1]. Herbert .W. Hethcote (2000). The Mathematics of Infectious Diseases. Society for Industrial and Applied Mathematics. Vol.42(4), 599-653.

[2]. Ibrahim, M.O. and Egbetade, S.A. (2012). A Mathematical Model for the Epidemiology of Tuberculosis with estimate of the basic reproduction number. Archimedes Journal Maths. Vol .2.

[3]. Jibril. L and Ibrahim, M.O. (2011). Mathematical Modeling on the CDTI Prospects for elimination of Onchocerciasis: A deterministic model Approach. Mathematics and Statistics. Vol.3(4), 136-140.

[4]. John, G. Ayisi, Anna. M.VanEijk, Robert D. Newman (2010). Maternal Malaria and Perinatal HIV Transmission, Western Kenya Centers for Disease Control and Prevention. Vol.4(3).

[5]. JulienArino, Connell. C. Mccluskey, and Van Den Driessche (2003). Global results for an epidemic model with Vaccination that exhibits Backward Bifurcation. Society for Industrialand Applied Mathematics. 64(1), 260-276.

[6]. Kakkilaya, B.S. (2012). Can Child Be Affected by Mother's Malaria. Malariasite.com.

[7]. Kakkilaya, B.S. (2011). Transmission of malaria. Malariasite.com

[8]. Kakkilaya, B.S. (2011). Pregnancy and Malaria. Malariasite.com

[9]. Labadin. J., C. Kon, M.L and Juan, S.F.S.(2009). Deterministic Malaria Transmission Model with Acquired Immunity. Proceedings of the World Congress on Engineering and Computer Science. Vol.2, 20-22

[10]. Lawi, G.O, Mugisha, J.Y.T and Omolo-ongati, N (2011). Mathematical Model for Malaria and Meningitis Co-infection among Children. Applied Mathematical Sciences. Vol.5 (47), 2337-2359.

[11]. Nakul R.C (2005). Using mathematical models in controlling the spread of malaria. Ph.D. thesis.

[12]. George, T. A (2012). A mathematical model to control the spread of malaria in Ghana 